\title{
Harmonic averages, exact difference schemes and local Green's functions in variable coefficient PDE problems
}

\author{
Owe Axelsson, ${ }^{\dagger}$, János Karátson $\ddagger$ \\ February 19, 2013
}

\begin{abstract}
A brief survey is given to show that harmonic averages enter in a natural way in the numerical solution of various variable coefficient problems, such as in elliptic and transport equations, also of singular perturbation types. Local Green's functions used as test functions in a Petrov-Galerkin finite element method combined with harmonic averages can be very efficient and are related to exact difference schemes.
\end{abstract}

Keywords: variable coefficients, harmonic averages, singular perturbation, local Green's functions, exact difference schemes.

\section{Introduction}

In many problems appearing in practice, partial differential equations with strongly variable (heterogeneous) coefficients enter. To enable an efficient discretization and iterative solution method, the coefficients should preferably be approximated by piecewise constants, i.e. constant on each element in a finite element mesh. Such an averaging can take place in various ways. In this paper we advocate the use of harmonic averages.

To demonstrate the natural appearance of such averages, we consider first an elementary example. Assume that a car driver passes $n$ parts on his road with different speed limits where some traffic conquestion can take place, reducing the possible velocity. For notational simplicity, let each such part of the road have equal length and let the velocity limits be $v_{i}, i=1,2, \ldots, n$. Assume, in addition, that the driver gets increasingly tired with time and gradually slows down his actual velocity below the allowed one. Let this be modeled by a decreasing function $0<f(t) \leq 1$. Then the actual velocity at time $t$ satisfies

$$
\frac{d x(t)}{d t}=v(x(t)) f(t), \quad t>0
$$

*Institute of Geonics AS CR, IT4 Innovations, Ostrava, The Czech Republic

$\dagger$ King Abdulaziz University, Jeddah, Saudi Arabia, owea@it.uu.se.

${ }^{\ddagger}$ Department of Applied Analysis and Computational Mathematics, ELTE University, and MTA-ELTE Numerical Analysis and Large Networks Research Group; Budapest, Hungary; karatson@cs.elte.hu. 
where $x(t)$ is the distance traveled at time $t$. Let $T$ be the time to the final destination where a distance $\ell$ has been traveled. Then

$$
\frac{1}{T} \int_{0}^{\ell} \frac{d x}{v(x)}=\frac{1}{T} \int_{0}^{T} f(t) d t
$$

that is, the average velocity during the whole journey is

$$
\frac{\ell}{T}=\bar{v} \frac{1}{T} \int_{0}^{T} f(t) d t
$$

where $\bar{v}=\frac{n}{\sum_{1}^{n} \frac{1}{v_{i}}}$ is the harmonic average of the velocities $\left\{v_{i}\right\}_{1}^{n}$. In a harmonic average, the smallest numbers tend to dominate. Hence, in this example, the slowest velocities tend to dominate.

As a numerical illustration, let $n=3$ and assume three different velocities, $v_{1}=$ $50, v_{2}=83.3$ and $v_{3}=125(\mathrm{~km} / \mathrm{h})$. Then $\bar{v}=75.0$. Further, let $\ell=300(\mathrm{~km})$ and assume that $f(t)=e^{-\alpha t}$, where $\alpha=0.05$ and time is measured in hours. Then

$$
\frac{\ell}{T}=\frac{75}{T} \int_{0}^{T} e^{-\alpha t} d t=\frac{75}{\alpha T}\left(1-e^{-\alpha T}\right)
$$

So

$$
e^{-\alpha T}=1-\frac{\alpha \ell}{75} \quad \text { or } \quad T=\alpha^{-1} \ln \left(1-\frac{\alpha \ell}{75}\right)^{-1}=20 \ln 5 / 4 \approx 4.45(h) .
$$

The actual average velocity is then $\frac{\ell}{T} \approx 67.4$. The arithmetic, harmonic and actual velocities are $86.1,75.0$ and $67.4 \mathrm{~km} / \mathrm{h}$, respectively.

Here harmonic averages of coefficients enter in a natural way.

The paper is organized as follows. Based on [11], in Section 2 first an elementary derivation for a $1 \mathrm{D}$ elliptic problem with variable coefficients is reviewed, and the use of harmonic averages is advocated for this but also for higher dimensional problems. Then we discuss a time-dependent convection-diffusion-reaction equation of singular perturbation type, where the diffusion coefficient is small relative to the other coefficients. Using moving grids along the characteristic lines defined by the convection part of the operator, one is led to an exact difference scheme.

Section 4 deals with the use of special finite element test functions. In many problems, local Green's functions can be used as very efficient finite element test functions. For singular perturbation type of problems there arise boundary and/or interior layers where the solution changes rapidly in narrow regions. Local Green's functions can be particularly useful in resolving those layers with no need to use very fine local meshes as is otherwise a common approach used to resolve the solution in the layers. In practice, however, for higher dimensional problems we must in general use approximate Green's functions. This study involves the practically important problem of Darcy flow and similar type of problems in porous media with highly heterogeneous coefficients. The paper ends with some remarks concerning the application of exact difference schemes to achieve a superlinear rate of convergence of a preconditioned conjugate gradient method for more general problem. 


\section{A brief summary of some schemes using harmonic averages}

Following [11] in this section we summarize some applications of harmonic averages .

\subsection{Variable coefficients in elliptic problems}

As an introduction to exact difference schemes, consider the 1D problem

$$
-\left(k(x) u^{\prime}(x)\right)^{\prime}=f(x), 0<x<1, u(0)=\alpha, u(1)=\beta \quad \text { where } k(x)>0,
$$

where we first study the homogeneous equation,

$$
-\left(k(x) u^{\prime}(x)\right)^{\prime}=0 .
$$

Here

$$
k(x) u^{\prime}(x)=c,
$$

where $c$ is an integration constant.

Let $\left[x_{i}, x_{i+1}\right]_{i=0}^{n-1}$, where $0=x_{0}<x_{1}<\ldots<x_{n}=1$, be a partition of the unit interval, and $u_{i}:=u\left(x_{i}\right)(i=0, \ldots, n)$. It follows from (3) that $u^{\prime}(x)=c / k(x)$ and

$$
u_{i+1}-u_{i}=c \int_{x_{i}}^{x_{i+1}} \frac{1}{k(x)} d x
$$

SO

$$
\bar{k}_{i+1 / 2} \frac{u_{i+1}-u_{i}}{x_{i+1}-x_{i}}=c
$$

where

$$
\bar{k}_{i+1 / 2}=\left(x_{i+1}-x_{i}\right) / \int_{x_{i}}^{x_{i+1}} \frac{1}{k(x)} d x .
$$

Therefore it holds

$$
\bar{k}_{i+1 / 2} \frac{u_{i+1}-u_{i}}{x_{i+1}-x_{i}}-\bar{k}_{i-1 / 2} \frac{u_{i}-u\left(x_{i-1}\right)}{x_{i}-x_{i-1}}=0, i=1,2, \ldots, n-1
$$

where $u_{0}=u(0), u_{n}=u(1)$.

It follows that this is an exact difference scheme, that is, $u_{i}$ equals the exact pointwise values of the solution of (1) at the nodepoints $\left\{x_{i}\right\}$. Note that the coefficient $\bar{k}_{i+1 / 2}$ equals the (integral) harmonic average of $k(x)$ on the interval $\left(x_{i}, x_{i+1}\right), i=0,1, \ldots, n-1$. We note also that $k$ may be allowed even to be unbounded here if $1 / k$ is integrable.

The above demonstrates that the harmonic averages of the variable coefficients can be a useful choice. As has been shown in [12], see also [11], there is, however, no such simple and practically useful relation for the inhomogeneous equation (1).

Instead we can then use the following approach. Let $\bar{k}(x)=\bar{k}_{i+1 / 2}, x_{i}<x<x_{i+1}, i=$ $0,1, \ldots, n-1$. To solve the averaged problem

$$
-\left(\bar{k}(x) \tilde{u}^{\prime}(x)\right)^{\prime}=f(x), 0<x<1
$$


we use the method of local Green's functions. This method will be described in Section 4 in a more general context. In the present situation the local Green's functions $g$ as test functions for equation (5) are in fact the standard piecewise linear hat functions, that is, the function $g$ at $x_{i}$ equals

$$
g_{x_{i}}(x)= \begin{cases}\frac{x-x_{i-1}}{x_{i}-x_{i-1}}, & x_{i-1} \leq x<x_{i} \\ \frac{x_{i+1}-x}{x_{i+1}-x_{i}}, & x_{i}<x \leq x_{i+1}\end{cases}
$$

To find the error, i.e. the difference between the solution $u$ of (1) and $\tilde{u}$ of (5), we use

$$
-\left(k(x) u^{\prime}(x)\right)^{\prime}=f(x)=-\left(\bar{k}(x) \tilde{u}^{\prime}(x)\right)^{\prime}=-\left(k(x) \tilde{u}^{\prime}(x)\right)^{\prime}+\left(\left((k(x)-\bar{k}(x)) \tilde{u}^{\prime}(x)\right)^{\prime} .\right.
$$

This shows that

$$
-\left(k(x)(u-\tilde{u})^{\prime}(x)\right)^{\prime}=\left((k(x)-\bar{k}(x)) \tilde{u}^{\prime}(x)\right)^{\prime} .
$$

Hence the error $u-\tilde{u}$ can be computed, at least approximately, by solving (6) numerically. In a variational form we have

$$
\int_{0}^{1} k(x)(u-\tilde{u})^{\prime} \cdot v^{\prime}(x) d x=\int_{0}^{1}(\bar{k}(x)-k(x)) \tilde{u}^{\prime}(x) v^{\prime}(x) d x
$$

for any $v \in H^{1}(0,1)$ being a member of the set of test functions.

Since $\bar{k}(x, \delta) \leq \underline{k}(x, \delta)$ on each subinterval $(x, x+\delta)$, where $\underline{k}$ denotes the arithmetic average $\underline{k}(x, \delta)=\frac{1}{\delta} \int_{x}^{x+\delta} k(x) d x$, it holds

$$
\int_{0}^{1} \bar{k}(x) v^{\prime}(x)^{2} d x \leq \int_{0}^{1} k(x) v^{\prime}(x)^{2} d x, \quad \forall v \in H^{1}(0,1),
$$

i.e., $\bar{k} \leq k$ in an energy sense. At this point we comment that for strongly variable coefficients it is less viable to use a numerical quadrature to solve (7). Instead, we can repeat the approach used, replacing $k(x)$ on the left hand side of (7) with $\bar{k}$ to compute a corresponding approximation of the error $u-\tilde{u}$. Possibly we can repeat this method in a defect-correction manner.

Here we are, however, more interested in what (4) can tell us about the choice of the averaged coefficients $\bar{k}$. Besides the harmonic integral average $\bar{k}$, we can use the arithmetic integral average $\underline{k}$, which satisfy $\bar{k} \leq \underline{k}$ or $\underline{k}^{-1} \leq \bar{k}^{-1}$. We can also take a convex combination, for which $\underline{k}^{-1} \leq \theta_{i} \bar{k}^{-1}+\left(1-\theta_{i}\right) \underline{k}^{-1} \leq \bar{k}^{-1}$ where $0 \leq \theta_{i} \leq 1$.

Assume that in some subdomain $\left[x_{i}, x_{i+1}\right]$ the coefficient $k$ takes few values near its maximum value and that most values are close to its minimal value. In this case an almost harmonic average of $k$, i.e. a value of $\theta_{i}$ close to 1 is a proper choice. If, however, the medium is stiffer, i.e. most values are close to its maximum, then we shall choose $\theta_{i}$ small.

Based on a knowledge of physical materials in each element, one can then make a proper choice for $\bar{k}$, e.g. close to a harmonic average or to an arithmetic average. The above analysis only applies for a 1D problem, but clearly, the method with averaged coefficient values is applicable also for higher dimensional problems. 
Since, in general, the local Green's functions are not known in explicit form for higher dimensional problems, it cannot be used straightforwardly. Based on the rapidly decaying shape of the Green's functions in 2D or 3D, one can, however, expect that the values closer to the node points play a greater role and, based on this, one can make a choice between a harmonic or arithmetic average for elements surrounding such a node point.

\subsection{Harmonic averages in the solution of time dependent convec- tion-reaction equations}

With the aim of later solving a singularly perturbed convection-diffusion-reaction equation, in this section we let the diffusion term be absent and consider the convectionreaction equation

$$
\begin{gathered}
\frac{\partial u}{\partial t}+a(x, t) \frac{\partial u}{\partial x}+b(y, t) \frac{\partial u}{\partial y}=f_{1}(x, y, t) f_{2}(u), \\
0<x<\ell_{1}, \quad 0<y<\ell_{2}, \quad t>0
\end{gathered}
$$

(where $a, b>0$ ) and with initial and boundary values

$$
\left.u\right|_{t=0}=u_{0}(x, y),\left.\quad u\right|_{x=0}=u_{1}(y, t),\left.u\right|_{y=0}=u_{2}(x, t) .
$$

The discussion follows [11].

We assume that the convection coefficients $a, b$ are separable functions in space and time and depend in space only on variables $x$ and $y$, respectively: i.e.,

$$
a(x, t)=a_{1}(x) a_{2}(t), \quad b(y, t)=b_{1}(y) b_{2}(t) .
$$

We will use a moving grid defined by the characteristic lines $\underline{\ell}(t)=(x(t), y(t))$ for the vector function defined by $(a, b)$, i.e.

$$
\frac{d x}{d t}=a_{1}(x) a_{2}(t), \quad \frac{d y}{d t}=b_{1}(y) b_{2}(t) .
$$

Since the vector field is uniquely defined, no two characteristic lines may cross each other. Using the chain rule, the derivative of $u$ along the characteristic lines satisfies

$$
\left.\frac{d u}{d t}\right|_{\underline{\ell}(t)}=\frac{\partial u}{\partial t}+\frac{\partial u}{\partial x} \frac{d x}{d t}+\frac{\partial u}{\partial y} \frac{d y}{d t}
$$

so the equation (8) along $\underline{\ell}(t)$ can be written as

$$
\left.\frac{d u}{d t}\right|_{\underline{\ell}(t)}=f_{1}(x, y, t) f_{2}(u) .
$$

This shows that when the characteristic lines have been computed, the solution of the convection-reaction equation can be computed as the solution of this initial value problem. 
We can now apply the same derivation as was used for the example in the introduction to rewrite the above equations in exact difference forms:

$$
\begin{gathered}
\frac{x_{i}^{n+1}-x_{i}^{n}}{\tau}=\left(\frac{1}{x_{i}^{n+1}-x_{i}^{n}} \int_{x_{i}^{n}}^{x_{i}^{n+1}} \frac{d x}{a_{1}(x)}\right)^{-1} \frac{1}{\tau} \int_{t_{n}}^{t_{n+1}} a_{2}(t) d t \\
\frac{y_{j}^{n+1}-y_{j}^{n}}{\tau}=\left(\frac{1}{y_{j}^{n+1}-y_{j}^{n}} \int_{y_{j}^{n}}^{y_{j}^{n+1}} \frac{d y}{b_{1}(y)}\right)^{-1} \frac{1}{\tau} \int_{t_{n}}^{t_{n+1}} b_{2}(t) d t \\
\frac{u_{i, j}^{n+1}-u_{i, j}^{n}}{\tau}=\left(\frac{1}{u_{i, j}^{n+1}-u_{i, j}^{n}} \int_{u_{i, j}^{n}}^{u_{i, j}^{n+1}} \frac{d u}{f_{2}(u)}\right)^{-1} \frac{1}{\tau} \int_{t_{n}}^{t_{n+1}} f_{1}(x(t), y(t), t) d t .
\end{gathered}
$$

Here $\tau=t_{n+1}-t_{n}$ is the timestep, and $(i, j)$ denotes a mesh point in a moving grid, defined by the characteristic lines, as illustrated in Figure 2.1.

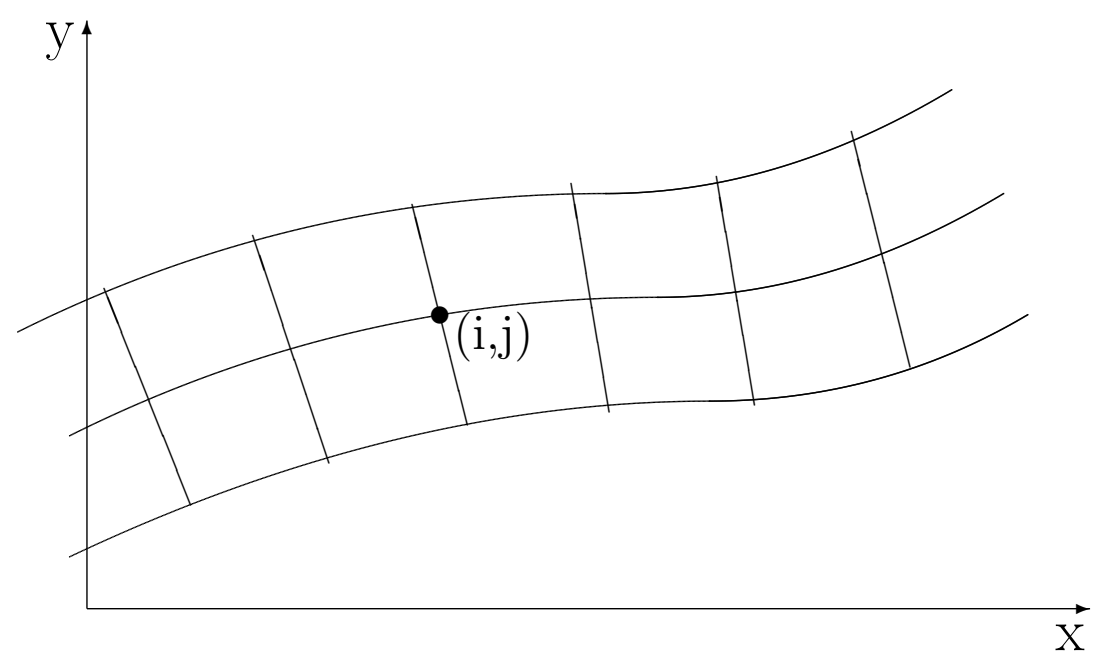

Figure 2.1. Part of a mesh defined for three characteristic lines.

It is seen that each of the equations (9), (10), (11) involve integral harmonic mean values of variable functions. Further, the equations are in general nonlinear, but only to a weak extent. This is due to two reasons. First, the nonlinear part is multiplied by a small factor, $\tau$. Second, the average values depend relatively little on the unknowns at time $t_{n+1}$. However, for general functions $a_{1}, b_{1}$ and $f_{2}$ intermediate unknown values enter. Normally, we then use some numerical quadrature, such as a Runge-Kutta method for the evaluation of the harmonic values. The unknown function values can then be approximated by corresponding interpolation polynomials.

For the numerical solution of the equations we must keep the variable coordinates $\left(x_{i}, y_{j}\right)$ stored, at least one time-step back. If we use, say, a quadratic interpolant to evaluate the integrals involving $a_{1}, b_{1}$ and $f_{2}$, we must keep the coordinates stored two time steps back. 
Consider now two examples of a reaction term in (11), where the integral can be evaluated exactly and there is no need to use any interpolation.

Example 2.1 Let $f_{2}(\cdot)$ be a linear function: $f_{2}(u)=-\lambda u, \lambda>0$. Then

$$
\left(u_{i j}^{n+1}-u_{i j}^{n}\right) / \int_{u_{i j}^{n}}^{u_{i j}^{n+1}} \frac{d u}{f_{2}(u)}=\frac{1}{\lambda}\left(u_{i j}^{n+1}-u_{i j}^{n}\right) / \ln \left(1+\frac{u_{i j}^{n}-u_{i j}^{n+1}}{u_{i j}^{n+1}}\right),
$$

which approximately equals $\frac{1}{\lambda} u_{i j}^{n+1}$ if $\left|u_{i j}^{n}-u_{i j}^{n+1}\right|$ is small relative to $u_{i j}^{n+1}$, i.e. if the time step is small. Hence the equation is nearly linear.

Example 2.2 Let $f_{2}(u)=-\lambda u^{2}, \lambda>0$.

Then

$$
\int_{u_{i j}^{n}}^{u_{i j}^{n+1}} \frac{d u}{f_{2}(u)}=\lambda\left(\frac{1}{u_{i j}^{n+1}}-\frac{1}{u_{i j}^{n}}\right) \text { and }\left(u_{i j}^{n+1}-u_{i j}^{n}\right) / \int_{u_{i j}^{n}}^{u_{i j}^{n+1}} \frac{d u}{f_{2}(u)}=-\frac{1}{\lambda} u_{i j}^{n+1} u_{i j}^{n} .
$$

In this case equation (11) is linear. This has been pointed out in [11].

For higher order power laws, where $f_{2}(u)=-\lambda u^{q}, \lambda>0, q>2$, we get

$$
\left(u_{i j}^{n+1}-u_{i j}^{n}\right) / \int_{n_{i j}^{u}}^{u_{i j}^{n+1}} \frac{d u}{f_{2}(u)}=-\frac{q-1}{\lambda} \frac{u_{i j}^{n+1}-u_{i j}^{n}}{\left(u_{i j}^{n+1}\right)^{q-1}-\left(u_{i j}^{n}\right)^{q-1}}\left(u_{i j}^{n+1} u_{i j}^{n}\right)^{q-1} .
$$

For $q=3$ this equals

$$
-\frac{2}{\lambda}\left(u_{i j}^{n+1} u_{i j}^{n}\right)^{2} /\left(u_{i j}^{n+1}+u_{i j}^{n}\right) .
$$

Again, this gives a nearly linear equation for small time steps, where $u_{i j}^{n+1} \approx u_{i j}^{n}$.

\section{On high order difference methods}

Now we turn to high order schemes which are related to exact difference schemes. We consider here difference methods for the Laplacian operator,

$$
\mathcal{L} u=f \text { in } \Omega, \quad u=0 \text { on } \partial \Omega,
$$

where $\mathcal{L}=\Delta=\frac{\partial^{2}}{\partial x^{2}}+\frac{\partial^{2}}{\partial y^{2}}$ and $\Omega$ is a rectangular domain.

For the familiar five-point and cross-directed five-point differences on a local equidistant square submesh it holds

$$
\begin{aligned}
& \Delta_{h}^{(5)}=h^{-2}\left[u_{h}(x-h, y)+u_{h}(x+h, y)+u_{h}(x, y-h)+u_{h}(x, y+h)-4 u_{h}(x, y)\right], \\
& \Delta_{h}^{(5, \times)}=\frac{1}{2} h^{-2}\left[u_{h}(x-h, y-h)+u_{h}(x+h, y-h)+\right. \\
& \left.u_{h}(x-h, y+h)+u_{h}(x+h, y+h)-4 u_{h}(x, y)\right], \quad(x, y) \in \Omega_{h} \text {. }
\end{aligned}
$$


It is readily seen that, for a sufficiently smooth function $u$,

$$
\begin{aligned}
\Delta_{h}^{(5)} & =\Delta u+\frac{2}{4 !} h^{2}\left(u_{x}^{(4)}+u_{y}^{(4)}\right)+\frac{2}{6 !} h^{4}\left(u_{x}^{(6)}+u_{y}^{(6)}\right)+O\left(h^{6}\right), \\
\Delta_{h}^{(5, \times)} & =\Delta u+\frac{2}{4 !} h^{2}\left(u_{x}^{(4)}+6 u_{x, y}^{(2,2)}+u_{y}^{(4)}\right)+\frac{2}{6 !} h^{4}\left(u_{x}^{(6)}+15 u_{x, y}^{(4,2)}+15 u_{x, y}^{(2,4)}+u_{y}^{(6)}\right)+O\left(h^{6}\right),
\end{aligned}
$$

where we use the readily understandable notation, for instance,

$$
u_{x, y}^{(4,2)}=u_{x x x x y y} \quad \text { etc. }
$$

The nine-point difference scheme is defined by

$$
\Delta_{h}^{(9)}=\frac{2}{3} \Delta_{h}^{(5)}+\frac{1}{3} \Delta_{h}^{(5, \times)} .
$$

The coefficients in this stencil equal $1 / 6$ for the corner vertex points in the square with edges $2 h, 2 / 3$ for the midedge points and $-10 / 3$ for the center points.

A computation shows that for a uniform rectangular mesh,

$$
\Delta_{h}^{(9)} u_{h}=f+\frac{1}{12} \Delta f+\frac{1}{360} h^{4}\left(\Delta^{2} f+2 f_{x x y y}\right)+O\left(h^{6}\right),
$$

where $\Delta^{2} f=\Delta(\Delta f)$. Using a modified right-hand side in the difference formula, it follows that the difference approximation

$$
\Delta_{h}^{(9)} u_{h}=\left[I+\frac{h^{2}}{12} \Delta_{h}^{(5)}\right] f, \quad(x, y) \in \Omega_{h},
$$

has truncation error $O\left(h^{4}\right)$.

Further, it follows from (12) that for a sufficiently smooth function $f$, we have $\Delta f=$ $\Delta_{h}^{(9)} f-\frac{1}{12} h^{2} \Delta^{2} f+O\left(h^{4}\right)$. A computation shows that $h^{2} f_{x, y}^{(2,2)}=2\left[\Delta_{h}^{(5, \times)} f-\Delta_{h}^{(5)} f\right]+O\left(h^{4}\right)=$ $6\left(\Delta_{h}^{(9)}-\Delta_{h}^{(5)}\right) f+O\left(h^{4}\right)$ and therefore, the nine-point stencil with the correspondingly modified right-hand side,

$$
\left.\Delta_{h}^{(9)} u_{h}=f+\frac{1}{12} h^{2} \Delta_{h}^{(9)} f-\frac{1}{240} h^{4} \Delta_{h}^{(5)}(\Delta f)\right)+\frac{1}{180} h^{4} f_{x, y}^{(2,2)}, \quad(x, y) \in \Omega_{h}
$$

has a truncation error $O\left(h^{6}\right)$. Here we may use the above approximation of the last term, which results in the difference scheme

$$
\Delta_{h}^{(9)} u_{h}=f+\frac{1}{6} h^{2}\left(\frac{1}{2}+\frac{1}{5} h^{2}\right) \Delta_{h}^{(9)} f-\frac{1}{30} h^{4}\left(\Delta_{h}^{(5)}\left(f+\frac{1}{8} \Delta f\right)\right) .
$$

The implementation of this scheme is simplified if $f$ is given analytically so that $\Delta f$ etc. can be computed explicitly. If $f \equiv 0$, then $\Delta_{h}^{(9)} \equiv 0$ has an order of approximation $O\left(h^{6}\right)$. Hence, this scheme provides a very accurate approximation, for instance for far field equations, where frequently $\Delta u=0$.

Since the difference scheme leads to an $M$-matrix, i.e. a matrix whose inverse has positive entries, it follows that the discretization error $u-u_{h}$ satisfies

$$
\max _{x \in \Omega_{h}}\left|u-u_{h}\right| \leq O\left(h^{6}\right) .
$$


Similar compact schemes, but with more mesh points have been published in [10]. As shown there and in [2] one can also derive difference methods of high order for anisotropic elliptic problems and with a mixed derivative term, and even with variable coefficients. For a practical evaluation of such methods for problems whose solutions have singularities or boundary or interior layers, see [9].

We now present high order difference methods based on hexagonal meshes. The corresponding seven point scheme is

$$
\Delta_{h}^{(7)} u_{h}=\left(\frac{h}{2}\right)^{2}\left[\frac{1}{6} \sum_{i=1}^{6} u_{h}\left(x_{i}, y_{i}\right)-u(x, y)\right],
$$

where $u\left(x_{i}, y_{i}\right), i=1,2 \ldots, 6$ are the hexagonal mesh nodepoints. A Taylor expansion shows that

$$
\Delta_{h}^{(7)} u_{h}=f+\frac{1}{4}\left(\frac{h}{2}\right)^{2}+\Delta f+\frac{1}{360}\left(\frac{h}{2}\right)^{4}\left(11 u_{x}^{(6)}+15 u_{x, y}^{(4,2)}+45 u_{x, y}^{(2,4)}+9 u_{y}^{(6)}\right)+O\left(h^{6}\right) .
$$

This scheme corresponds to a horizontal ordering of the hexagonal mesh. Similarly, for a 90 degree oriented scheme, one gets

$$
\Delta_{h}^{(7, \times)} u_{h}=f+\frac{1}{4}\left(\frac{h}{2}\right)^{2} \Delta f+\frac{1}{360}\left(\frac{h}{2}\right)^{4}\left(9 u_{x}^{(6)}+45 u_{x, y}^{(4,2)}+15 u_{x, y}^{(2,4)}+11 u_{y}^{(6)}\right)+O\left(h^{6}\right) .
$$

One can solve the difference approximations

$$
\begin{aligned}
& \Delta_{h}^{(7)} u_{h}^{(1)}=f+\frac{1}{4}\left(\frac{h}{2}\right)^{2} \Delta f+\frac{1}{36}\left(\frac{h}{2}\right)^{4} \Delta^{2} f, \\
& \Delta_{h}^{(7, \times)} u_{h}^{(2)}=f+\frac{1}{4}\left(\frac{h}{2}\right)^{2} \Delta f+\frac{1}{36}\left(\frac{h}{2}\right)^{4} \Delta^{2} f
\end{aligned}
$$

separately and take the average $\frac{1}{2}\left(u_{h}^{(1)}+u_{h}^{(2)}\right)$ of the solutions or, alternatively, solve

$$
\frac{1}{2}\left(\Delta_{h}^{(7)}+\Delta_{h}^{(7, \times)}\right) u_{h}=f+\frac{1}{4}\left(\frac{h}{2}\right)^{2} \Delta f+\frac{1}{36}\left(\frac{h}{2}\right)^{4} \Delta^{2} f
$$

to get an $O\left(h^{6}\right)$ truncation error. Here

$$
\begin{aligned}
\Delta^{2} f & =\left(\frac{\partial^{2}}{\partial x^{2}}+\frac{\partial^{2}}{\partial y^{2}}\right)^{3} u \\
& =u_{x}^{(6)}+3 u_{x, y}^{(4,2)}+3 u_{x, y}^{(2,4)}+u_{y}^{(6)} .
\end{aligned}
$$

Clearly, as before, we can replace $\Delta f$ with $\frac{1}{2}\left(\Delta_{h}^{(7)}+\Delta_{h}^{(7, x)}\right)$, to enable pointwise computations of $\Delta f$ and avoiding the need to compute 4th order derivatives of $f$.

It can be seen that the error coefficient is significantly smaller for the hexagonal mesh difference method. However, for a hexagonal mesh one may have to use special boundary approximations. This can be done. e.g. by use of, possibly high order, finite element approximations at boundary elements. We do not discuss this problem further here. In some problems, the solution near the boundary is practically zero, in which case there is no need to use such approximations. 


\section{Applications of local Green's functions}

We first recall some basic properties of local Green's functions, used as finite element basis functions. We give its main idea with a simple example of a uniform grid approximation. Let

$$
u_{h}(x, y)=\sum_{k=1}^{N} u_{k} \varphi_{k}(x, y)
$$

be an approximation of the exact solution of the partial differential equation $\mathcal{L} u=f$ in terms of basis functions $\varphi_{k}=\varphi\left(\left(x-x_{k}\right) / h,\left(y-y_{k}\right) / h\right)$ defined at the interior nodes $\left(x_{k}, y_{k}\right)$ of a grid covering $\Omega$ with a step $h$. Here $\varphi(x, y)$ is a chosen shape-function. In line with the Petrov-Galerkin method, the unknown coefficients $u_{k}$ are determined from the variational condition

$$
\left(\mathcal{L} u_{h}-f, \psi_{l}\right)=0, \quad l=1,2, \ldots, N
$$

for suitably chosen test functions.

Using the adjoint operator $\hat{\mathcal{L}}$ for which $(\mathcal{L} u, v)=(u, \hat{\mathcal{L}} v)$, the variational equality (14) can be rewritten in the form

$$
\left(u_{h}-u, \hat{\mathcal{L}} \psi_{l}\right)=0
$$

For an equation where $\mathcal{L}=-\varepsilon \Delta u+\underline{b} \cdot \underline{\nabla} u+c u$ the operator $\hat{\mathcal{L}}$ has the form

$$
\hat{\mathcal{L}} u \equiv-\varepsilon \Delta u-\nabla \cdot(\underline{b} u)+c u \text {. }
$$

If the shape-function $\psi$ complies with the equality $\hat{\mathcal{L}} \psi=\delta$, where $\delta(x, y)$ is Dirac's delta-function, then it follows from (15) that the approximate and the exact solutions coincide at the nodes, i.e. $u_{h}\left(x_{l}, y_{l}\right)=u\left(x_{l}, y_{l}\right)$ at the nodes.

In general, we can require $\hat{\mathcal{L}} \psi=\delta$ to hold locally on the supports $\omega_{l}$ of the test functions $\psi_{l}$. For a uniform grid, the projectors $\psi_{l}$ can be localized in $2 h \times 2 h$ squares centered at the nodes (Figure 4.1), i.e. in such a case $\omega=\omega_{h}$ is a square $|x| \leq h,|y| \leq h$. In accordance with the general scheme, $\psi$ must satisfy the Dirichlet boundary condition

$$
\left.\psi\right|_{\Gamma_{h}}=0
$$

where $\Gamma_{h}$ is the boundary of $\omega_{h}$. The shape-function $\psi(x, y)$ is the local Green's function of the problem considered. 


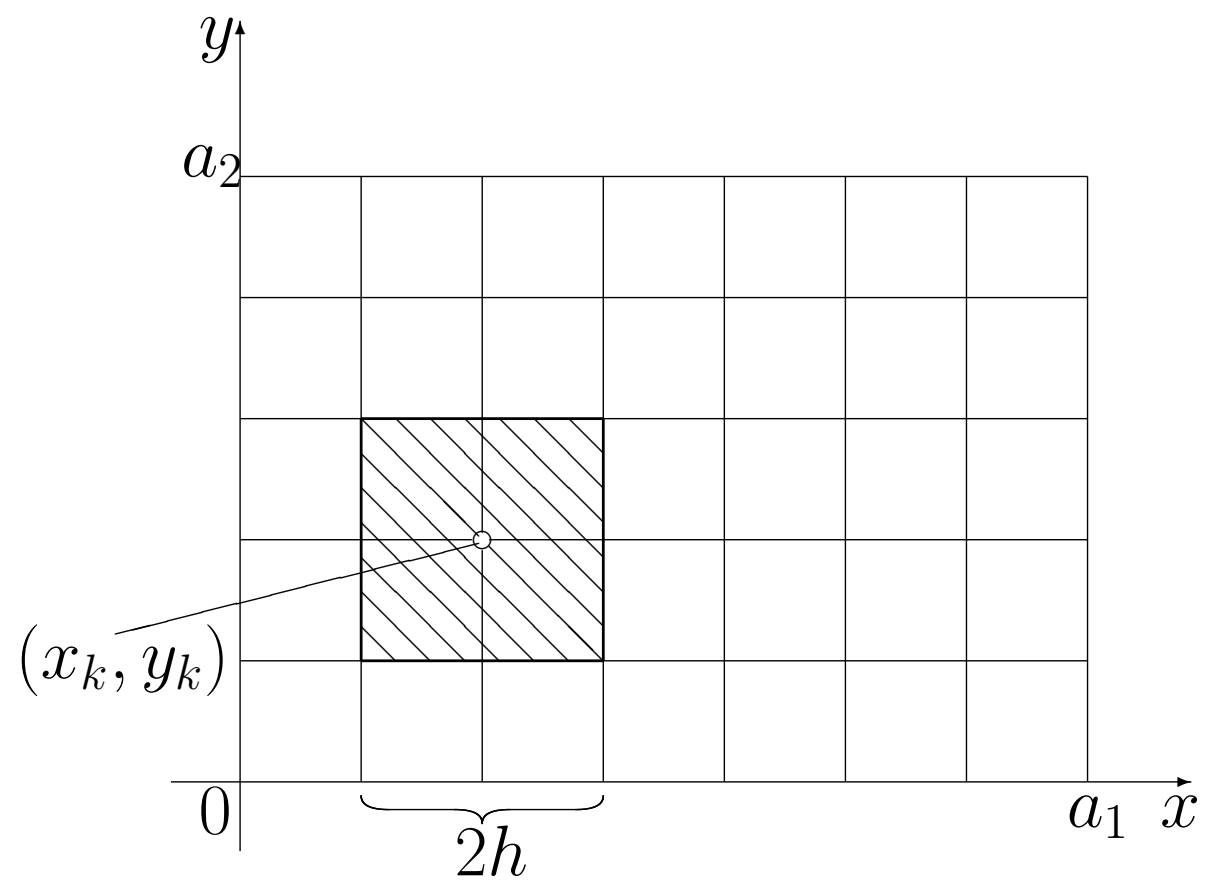

Figure 4.1.

In practice the local Green's function method exhibits high accuracy at the nodes even with a coarse grid. It is further of significant importance that appearance of the layers as $\varepsilon \rightarrow 0$ does not degrade its numerical stability. Besides the theoretical heuristic calculations above, this fact has been shown numerically as well, however mostly only for 1D examples $[1,8]$. Extension of the method to several variables has been restrained by the absence of an analytical solution of the Green's function, but suitable realization has been elaborated in $[3,4]$.

\subsection{A singularly perturbed equation}

We briefly describe a motivating example where one benefits by using local Green's functions. Consider the equation

$$
\frac{\partial u}{\partial t}+\mathcal{L}_{\varepsilon} u:=\frac{\partial u}{\partial t}-\varepsilon \Delta u+\left(b_{1}, b_{2}\right) \cdot \underline{\nabla} u=f(u, x, y, t), t>0
$$

in the unit square $\Omega=\{(x, y), 0<x<1,0<y<1\}$. We assume Dirichlet boundary conditions on $\partial \Omega$. The second order diffusion term, added to (8), where $0<\varepsilon \ll 1$, causes sharp boundary and/or interior layers in the solution. Since, by assumption, $b_{1}>0$, $b_{2}>0$, i.e. doesn't change sign in the interior of $\Omega$, in this example only boundary layers can appear. For small values of $\varepsilon$ and fairly smooth solutions of the reduced equation (where $\varepsilon=0$ ) in Section 3, this solution is close to the solution of (17), except in the boundary layers. The boundary layers arise due to that, in general, the reduced equation has a solution which does not satisfy the boundary conditions on the outflow boundary parts

$$
x=1,0<y<1 \text { and } y=1,0<x<1
$$

of $\partial \Omega$. Hence boundary layers occur there.

A common numerical approach is to resolve the boundary layers by use of very fine local meshes, with a mesh size a fraction of $\varepsilon$. This causes complications and very large sized 
linear systems to be solved. Instead, here we follow [4], see also [3], and advocate the use of local Green's functions. To illustrate this, consider first the 1D singular perturbation equation,

$$
-\varepsilon u^{\prime \prime}+a u^{\prime}=f, 0<x<1, u(0)=\alpha, u(1)=\beta
$$

where $a>0$ is constant. Then there appears a layer at $x=1$.

The local Green's function at a point $x_{0} \in(0,1)$ in the interval $\left[x_{0}-h, x_{0}+h\right]$ satisfies

$$
-\varepsilon g_{x_{0}}^{\prime \prime}-a g_{x_{0}}^{\prime}=\delta_{x_{0}}, \quad x_{0}-h<x<x_{0}+h
$$

where $\delta_{x_{0}}$ is the Dirac's function at $x_{0}$. It follows that

$$
g_{x_{0}}(x)= \begin{cases}\frac{1-e^{-\frac{a}{\varepsilon}\left(x-x_{0}+h\right)}}{1-e^{-\frac{a h}{\varepsilon}}}, & x_{0}-h<x \leq x_{0} \\ \frac{e^{-\frac{a}{\varepsilon}\left(x-x_{0}\right)}-e^{-\frac{a}{\varepsilon} h}}{1-e^{-\frac{a}{\varepsilon} h}}, & x_{0}<x<x_{0}+h .\end{cases}
$$

For our 2D problem, we can use this shape of the Green's function along the corresponding part of the characteristic line though a point $x_{0}, y_{0}$ and use piecewise linear basis functions along its orthogonal direction. In practice we use normally local Green's functions only in the boundary layer or even only at points where the layer occurs and standard basis functions elsewhere.

It is possible to solve the equation $\frac{\partial u}{\partial t}+\mathcal{L}_{\varepsilon} u=f$ by use of a defect-correction method. Given some initial approximation $u^{0}$ we compute then first the residual, $r^{0}=f-\frac{\partial u^{0}}{\partial t}+$ $\mathcal{L}_{\varepsilon} u^{0}$ and then a correction, $\delta u^{0}$ by solving the reduced equation $\frac{\partial \delta u^{0}}{\partial t}+\mathcal{L}_{0}\left(\delta u^{0}\right)=r^{0}$ in $\Omega$ and the corresponding correction at all boundary layers by use of the local Green's function basis functions.

\subsection{Elliptic problems with heterogeneous coefficients}

As an example of a problem where heterogeneous coefficients frequently appear, we consider a Darcy flow problem

$$
\underline{v}=-K \nabla p \quad \text { in } \Omega
$$

where $\Omega$ is a bounded domain. For simplicity, we deal only with $2 \mathrm{D}$ bounded and connected domains. Here $\underline{v}$ denotes a velocity field vector function and $p$ denotes the pressure variable. Normally $\underline{v}$ satisfies a constraint equation, like $\underline{\nabla} \cdot \underline{v}=f$ for a given source function $f$. For consistency, we assume that $\int_{\Omega} f=0$. This leads to the coupled problem

$$
\begin{cases}\underline{v}+K \nabla p & =0 \\ \nabla \cdot \underline{v} & =f\end{cases}
$$

Such coupled equations arise more generally if we use a mixed variable formulation for the elliptic equation,

$$
\mathcal{L} u:=-\underline{\nabla} \cdot(K \nabla u)=f \quad \text { in } \Omega \subset \Re^{2}
$$

where $K$ is a symmetric, positive definite matrix of order $2 \times 2$. Proper boundary conditions hold for $u$. The equation (18) is equally applicable in heat and electric conduction 
problems where $u$ denotes the temperature and voltage and $K$ the thermal and electric conductivity, respectively. It appears in other applications as well.

In many applications, such as in groundwater flow, $K$ can vary greatly between narrow regions and can even have an oscillatory behaviour, where the ratio between its maximal and minimal amplitudes can be very large. If we denote $\varepsilon=\min \|K\| / \max \|K\|$ in a subdomain $\omega$ and $\varepsilon \ll 1$ there, then the problem can be characterized as a singularly perturbed problem exhibiting sharp interior and boundary layers, such as the example in Section 4.1.

Now, however, there can occur many interior layers. As is well known, standard Galerkin finite element methods, used on a coarse mesh with mesh parameter $H>\varepsilon$, give approximate solutions with unphysical oscillations in layers which, furthermore, spread out away from the layer subdomains and are hence practically useless. On the other hand, if one uses a mesh with mesh size $h \ll \varepsilon$ to resolve the solution at the layers, one is forced to solve extremely large scale problems when $\varepsilon \ll 1$. They can be too large even for presently available supercomputers.

Instead we shall use a fairly coarse mesh $\Omega_{H}$ with $H \gg \varepsilon$, but will then first replace matrix $K$ with a piecewise constant matrix $\bar{K}$, which is constant in each element of $\Omega_{H}$. Then we can use a standard Galerkin variational method to numerically solve the corresponding problem

$$
-\underline{\nabla} \cdot(\bar{K} \nabla u)=f \quad \text { on } \Omega_{H} .
$$

However, for similar reasons as before, we will instead use a Petrov-Galerkin method with local Green's functions as test functions. Either way, now there will occur no unphysical oscillations in the so computed approximate solution $u_{H}$, but this solution is in general smeared out in the layers and it cannot show any fine details of the exact solution.

To compute better approximations, we compute then first the residual $r_{h}=f-\mathcal{L} u_{H}$. Where $\left\|r_{h}\right\|$ is too large, say in some a subdomain $\omega_{H}$, we make then a local refinement of the mesh and compute corrections $\delta u_{h}$ by solving the corresponding local problems $\mathcal{L}\left(\delta u_{h}\right)=r_{h}$, using a local finer mesh on all such subdomains $\omega_{h}$. The method can be repeated in a defect-correction manner.

Essential parts of the method are how we compute the local averages and test basis functions. As we have seen in Section 2, harmonic averages and local Green's functions enter in a natural way. This will also be the basis for the approach taken here. Consider then problem (18) in a square domain $\Omega=[0,1]^{2}$ with, for simplicity, Dirichlet boundary conditions

$$
\left.u\right|_{\partial \Omega}=0 .
$$

The domain is partitioned uniformly in triangular elements. On each element $\left(e_{\ell}\right)$ we compute integral harmonic averages $\bar{K}$ of $K$, where

$$
\bar{K}=\left(\frac{1}{\left|e_{\ell}\right|} \int_{e_{\ell}} K^{-1} d \underline{x}\right)^{-1},
$$

where $\left|e_{\ell}\right|$ denotes the area of $e_{\ell}$. This can be done using some proper numerical quadrature rule. 
As remarked in subsection 2.1, we can alternatively use an arithmetic integral average or a convex combination of harmonic and arithmetic averages. For stiffer materials, the combination should be close to an arithmetic average.

We will use nodepoints corresponding to piecewise linear finite element basis functions.

For simplicity of notation, from now on we denote the points in the plane by $\mathbf{x}$ instead of $(x, y)$, and in particular, the complete set of nodepoints will be denoted by $\mathbf{x}_{1}, \ldots, \mathbf{x}_{N}$. Then the basis functions satisfy

$$
\sum_{k=1}^{N} \varphi_{k} \equiv 1 \quad \text { and } \quad \varphi_{k}\left(\mathbf{x}_{\ell}\right)=\delta_{k \ell} \quad(k, \ell=1, \ldots, N)
$$

where $\delta_{k \ell}$ is the Kronecker symbol.

We introduce as test functions the local Green's functions $\psi_{\ell}$ corresponding to the nodepoints $\mathbf{x}_{\ell}$, i.e., if $\omega_{\ell}$ denotes the patch around $\mathbf{x}_{\ell}$ (the support of the basis function with nodepoint $\mathbf{x}_{\ell}$ ) and $\Gamma_{\ell}$ denotes its boundary, then $\psi_{\ell}$ is the solution of

$$
\left\{\begin{array}{l}
\mathcal{L} \psi_{\ell}=\delta_{\ell} \quad \text { in } \omega_{\ell} \\
\psi_{\ell \mid \Gamma_{\ell}}=0
\end{array}\right.
$$

where $\delta_{\ell}$ is Dirac's delta-function at $\mathbf{x}_{\ell}$. Further, we extend $\psi_{\ell}$ to $\Omega$ by

$$
\psi_{\ell \mid \Omega \backslash \Gamma_{\ell}} \equiv 0
$$

Multiplying (22) with a function $v \in H_{0}^{1}(\Omega)$ and integrating, we obtain

$$
\int_{\omega_{\ell}} \bar{K} \nabla \psi_{\ell} \cdot \nabla v-\int_{\Gamma_{\ell}}\left(\partial_{\nu_{K}} \psi_{\ell}\right) v=v\left(\mathbf{x}_{\ell}\right)
$$

where $\partial_{\nu_{K}} \psi_{\ell}=\bar{K} \nu \cdot \nabla \psi_{\ell}$ denotes the weighted normal derivative of $\psi_{\ell}$.

We note that the $\psi_{\ell}$ cannot be given in closed form, hence in practice one has to compute them numerically. However, since (22) are local problems, they can be solved very accurately with some high order FEM such that the arising error is negligible w.r.t. the error of our original problem, and hence we will further in Section 3.4 consider this error as zero, see also Remark 4.1. In the next section we present a method to get such an accurate approximation. 


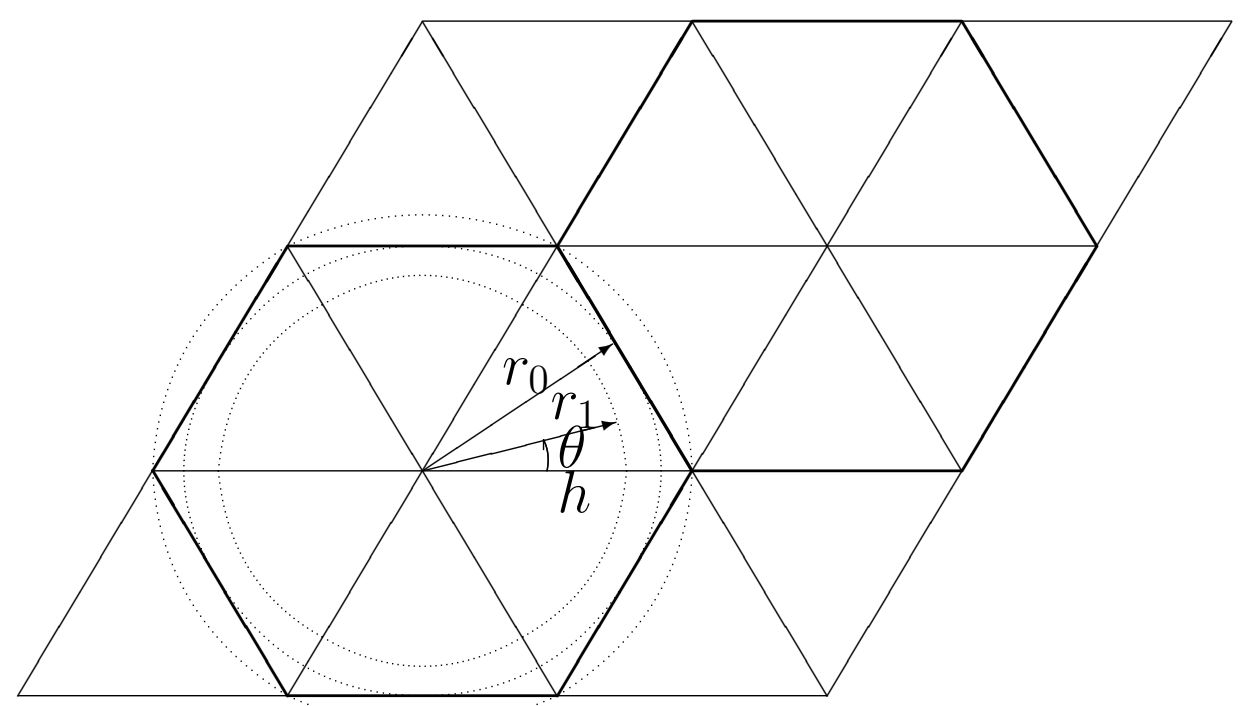

Figure 4.2 Hexagonal elements and polynomially extended radial basis functions $r_{1}=r_{0}-\left(h-r_{0}\right)$

\subsection{Approximation based on hexagonal meshes}

We present now two approaches to handle the construction of approximate Green's functions.

Consider a hexagonal mesh as given in Figure 4.2. We assume that any discontinuities in the coefficients $K$ take place only across edges, that is $K$ is assumed to be constant in each triangle.

The first approach is based on the use of radial basis functions $\tilde{\psi}_{l}(r)=\ln \frac{h}{r}, 0<r<h$, i.e. on the exact Green's functions in each nodepoint and integration over the disks with radius $h$. Thereby, for the evaluation of the line integrals in (22) we must use basis functions for $v$ in each nodepoint in the triangles where we integrate. If we use linear basis functions, besides the basis functions in the six nodepoints in each hexagon, we needs basis function in the six node points in the triangles outside the hexagon, see Figure 4.2 where we have marked four of those. This gives a less sparse matrix to handle, but is doable.

Another problem arises since the polynomial basis functions for the solution do not behave polynomially on the segments of the circle. Although the normal derivative of $\tilde{\psi}_{l}$ is constant on the circle edges, we must therefore use numerical integration on the edges, i.e. divide it in smaller segments and use an integration role, such as the trapezoidal rule, or better use a higher order Runge-Kutta method, such as based on Lobatto quadrature points. In the latter case very few integration points are needed.

A more severe complication arises for hexagons next to the outer boundary $\partial \Omega$ since part of the integration circles will lie outside the domain $\Omega$. A similar problem arises when we have discontinuous coefficients inside the circle.

We are then forced to use test basis functions with support inside the hexagon. One way to construct such replacements is the following. This approximation can in fact be applied in all hexagons. In this way we have sparser matrices with only six nonzero entries outside the main diagonal. 
We show this construction for a triangle as illustrated in Figure 3.3. The approximate Green's function is here based on part of the radial basis function up to a disc with radius $r_{1}=h / 2$. The use of the radial basis functions around the center nodepoint accounts for the singular behaviour of the exact Green's function. The radial basis function is then extended to the edge of the triangles with a polynomial of degree $q$, having the same first $q-1$ derivatives as the radial basis function at the edge of the inner disc.

Let $\xi(r)=\left(\frac{r}{r_{1}}-1\right) / a$, where $a=\frac{r_{0}}{r_{1} \cos \theta}-1$. Then $\xi\left(r_{1}\right)=0, \xi\left(\frac{r_{0}}{\cos \theta}\right)=1$ and $\xi^{\prime}(r)=\frac{1}{r_{1} a}$. Let now $g(r, \theta)$ be a polynomial of degree $q$ along a radius from a nodepoint, such that

$$
g\left(r_{1}, \theta\right)=\psi\left(r_{1}, \theta\right)=\ln \frac{h}{r_{1}}, \quad g\left(\frac{r_{0}}{\cos \theta}, \theta\right)=0 .
$$

and

$$
\frac{\partial^{\ell} g}{\partial r^{\ell}}\left(r_{1}, \theta\right)=\frac{\partial^{\ell}}{\partial r^{\ell}} \psi\left(r_{1}, \theta\right)=\left(-\frac{1}{r_{1}}\right)^{\ell}, \quad \ell=1,2, \ldots, q-1 .
$$

We let $g$ take the form

$$
g(r, \theta)=\psi\left(r_{1}, \theta\right)\left(1-\xi^{q}\right)+(1-\xi) \sum_{k=0}^{q-1} \frac{c_{k}}{k !} \xi^{k},
$$

where $c_{0}=0$. It follows that $g\left(r_{1}, \theta\right)=\psi\left(r_{1}, \theta\right)$ and $g\left(\frac{r_{0}}{\cos \theta}, 0\right)=0$. Further, a computation shows that

$$
\begin{aligned}
& g^{\ell}(r, \theta)=\frac{\partial^{\ell}}{\partial r^{\ell}} g(r, \theta)=-\psi\left(r_{1}, \theta\right) \frac{q !}{(q-\ell) !} \xi^{q-\ell}\left(\xi^{\prime}\right)^{\ell} \\
& \quad+\left(\xi^{\prime}\right)^{\ell} \sum_{k=\ell}^{q-1} \frac{c_{k}}{(k-\ell) !} \xi^{k-\ell}-\left(\xi^{\prime}\right)^{\ell} \sum_{k=\ell-1}^{q-1}(k+1) \frac{c_{k}}{(k-\ell+1)} \xi^{k-\ell+1}
\end{aligned}
$$

Hence at $r=r_{1}$ ( i.e. $\left.\xi(r)=0\right)$ it holds

$$
\left(-\frac{1}{r_{1}}\right)^{\ell}=g^{(\ell)}\left(r_{1}, \theta\right)=\left(\xi^{\prime}\right)^{\ell}\left(c_{l}-\ell c_{\ell-1}\right)=\left(\frac{1}{a r_{1}}\right)^{\ell}\left(c_{l}-\ell c_{\ell-1}\right),
$$

that is,

$$
c_{\ell}=\ell c_{\ell-1}+(-a)^{\ell}, \quad \ell=1,2, \ldots, q-1 .
$$

At $\xi=1$, where the boundary integral will be evaluated, $g^{\prime}(r, \theta)$ takes the form

$$
\begin{aligned}
g^{\prime}\left(\frac{r_{0}}{\cos \theta}, \theta\right) & =-\psi\left(r_{1}, \theta\right) q+\xi^{\prime}\left(\sum_{k=1}^{q-1} \frac{c_{k}}{(k-1) !}-\sum_{k=1}^{q-1}(k+1) \frac{c_{k}}{k !}\right) \\
& =-\frac{q}{a r_{1}} \ln \frac{h}{r_{1}}-\frac{1}{r_{1}} \sum_{k=1}^{q-1} \frac{c_{k} / a}{k !}
\end{aligned}
$$

Using (24), this expression can be simplified as follows. It holds, 


$$
\begin{aligned}
\frac{c_{q} / a}{(q-1) !} & =\frac{c_{q-1} / a}{(q-2) !}-\frac{(-a)^{q-2}}{(q-1) !} \\
2 \frac{c_{q-1} / a}{(q-2) !} & =2 \frac{c_{q-2} / a}{(q-3) !}-2 \frac{(-a)^{q-3}}{(q-2) !} \\
\frac{c_{q-\ell+1} / a}{(q-\ell) !} & =\ell \frac{c_{q-\ell} / a}{(q-\ell-1) !}-\ell \frac{(-a)^{q-\ell-1}}{(q-\ell) !} \\
(q-2) \frac{c_{2} / a}{2 !} & =(q-2) \frac{c_{1} / a}{1}-(q-2) \frac{(-a)}{2 !} \\
(q-1) c_{1} / 2 & =-(q-1) .
\end{aligned}
$$

Hence,

$$
\begin{aligned}
-\sum_{k=1}^{q-1} \frac{c_{k} / a}{k !} & =q-1+\sum_{2}^{q-1}(q-k) \frac{(-a)^{k-1}}{k !} \\
& =q-1-\frac{q}{a} \sum_{2}^{q-1} \frac{(-a)^{k}}{k !}-\sum_{2}^{q-1} \frac{(-a)^{k-1}}{(k-1) !} \\
& =-\frac{q}{a} \sum_{1}^{q-1} \frac{(-a)^{k}}{k !}-\sum_{k=0}^{q-2} \frac{(-a)^{k}}{k !} .
\end{aligned}
$$

We evaluate now this expression for a polynomial of degree $q=3$, to get

$$
-\frac{3}{a}\left(\frac{1}{2} a^{2}-a\right)-1+a=-\frac{1}{2} a+2 \text {. }
$$

Hence

$$
g^{\prime}\left(\frac{r_{0}}{\cos \theta}, \theta\right)=-\frac{1}{r_{1}}\left(\frac{3}{a} \ln 2+\frac{a}{2}-2\right)
$$

where $a=\frac{r_{0}}{r_{1} \cos \theta}-1=\frac{\sqrt{3}}{\cos \theta}-1$. Here $0<|\theta|<\pi / 6$ so $\frac{\sqrt{3}}{2} \leq \cos \theta \leq 1$.

For the evaluation of the boundary integral terms along the triangular edges one uses the normal derivative factor, $g^{\prime}\left(\frac{r_{0}}{\cos \theta}, \theta\right) \cos \theta$, multiplied by the interpolant of the solution.

\subsection{Error estimates based on exact local Green's functions}

The weak form of (19) with the test function $\psi_{\ell}$ for the exact solution $u$ and for the FEM solution $u_{h}$ yields

$$
\int_{\omega_{\ell}} \bar{K} \nabla u \cdot \nabla \psi_{\ell}=\int_{\omega_{\ell}} f \psi_{\ell} \quad \text { and } \quad \int_{\omega_{\ell}} \bar{K} \nabla u_{h} \cdot \nabla \psi_{\ell}=\int_{\omega_{\ell}} f \psi_{\ell}
$$

respectively. Subtracting these and using (23) for $v:=u-u_{h}$, we obtain the following formula:

$$
0=\int_{\omega_{\ell}} \bar{K} \nabla\left(u-u_{h}\right) \cdot \nabla \psi_{\ell}=\int_{\Gamma_{\ell}}\left(\partial_{\nu_{K}} \psi_{\ell}\right)\left(u-u_{h}\right)+u\left(\mathbf{x}_{\ell}\right)-u_{h}\left(\mathbf{x}_{\ell}\right) .
$$

That is, we have verified 
Corollary 4.1 The exact and FEM solutions and the local Green's functions are related as

$$
u\left(\mathbf{x}_{\ell}\right)-u_{h}\left(\mathbf{x}_{\ell}\right)=-\int_{\Gamma_{\ell}}\left(\partial_{\nu_{K}} \psi_{\ell}\right)\left(u-u_{h}\right)
$$

The FEM solution can be written as $u_{h}(\mathbf{x})=\sum_{k=1}^{N} c_{k} \varphi_{k}(\mathbf{x})$. Here (21) implies $c_{k}=$ $u_{h}\left(\mathbf{x}_{k}\right)$ for all $k$, hence

$$
u_{h}(\mathbf{x})=\sum_{k=1}^{N} u_{h}\left(\mathbf{x}_{k}\right) \varphi_{k}(\mathbf{x}) \quad(k=1, \ldots, N) .
$$

We also define the interpolants of $u$ by

$$
u_{i n t}(\mathbf{x})=\sum_{k=1}^{N} u\left(\mathbf{x}_{k}\right) \varphi_{k}(\mathbf{x}) \quad(k=1, \ldots, N),
$$

which satisfy

$$
u_{\text {int }}\left(\mathbf{x}_{k}\right)=u\left(\mathbf{x}_{k}\right) \quad(k=1, \ldots, N),
$$

i.e. the $u_{\text {int }}$ are (unknown) combinations of the basis functions being exact at the nodepoints.

In what follows, we study the maximum of the nodal errors defined by

$$
e\left(\mathbf{x}_{\ell}\right):=u\left(\mathbf{x}_{\ell}\right)-u_{h}\left(\mathbf{x}_{\ell}\right)
$$

or, by (26),

$$
e\left(\mathbf{x}_{\ell}\right)=u_{\text {int }}\left(\mathbf{x}_{\ell}\right)-u_{h}\left(\mathbf{x}_{\ell}\right) \quad(\ell=1, \ldots, N) .
$$

We start from Corollary 4.1, which yields

$$
e\left(\mathbf{x}_{\ell}\right)=-\int_{\Gamma_{\ell}}\left(\partial_{\nu_{K}} \psi_{\ell}\right)\left(u-u_{h}\right)
$$

Then

$e\left(\mathbf{x}_{\ell}\right)=-\int_{\Gamma_{\ell}}\left(\partial_{\nu_{K}} \psi_{\ell}\right)\left(u-u_{i n t}\right)-\int_{\Gamma_{\ell}}\left(\partial_{\nu_{K}} \psi_{\ell}\right)\left(u_{i n t}-u_{h}\right)=-\int_{\Gamma_{\ell}}\left(\partial_{\nu_{K}} \psi_{\ell}\right)\left(u-u_{i n t}\right)-\int_{\Gamma_{\ell}}\left(\partial_{\nu_{K}} \psi_{\ell}\right) e$,

letting here

$$
e(\mathbf{x}):=u_{\text {int }}(\mathbf{x})-u_{h}(\mathbf{x})
$$

now denote the error function for all $\mathrm{x} \in \Omega$.

Proposition 4.1 Let $\ell \in\{1, \ldots, N\}$. If there exists $q<1$ such that

$$
\left|\int_{\Gamma_{\ell}}\left(\partial_{\nu_{K}} \psi_{\ell}\right) e\right| \leq q\left|e\left(\mathbf{x}_{\ell}\right)\right|
$$

then

$$
\left|e\left(\mathbf{x}_{\ell}\right)\right| \leq \frac{1}{1-q}\left|\int_{\Gamma_{\ell}}\left(\partial_{\nu_{K}} \psi_{\ell}\right)\left(u-u_{i n t}\right)\right| .
$$


PROOF. It is an obvious rearrangement of (28).

We are interested in estimating the maximal error $\max _{\ell}\left|e\left(\mathbf{x}_{\ell}\right)\right|$. We check then how condition (29) can be satisfied, i.e. how we can bound the integral in its l.h.s.,

$$
\int_{\Gamma_{\ell}} \partial_{\nu_{K}} \psi_{\ell}(s) e(s) d s \quad\left(s \in \Gamma_{\ell}\right) .
$$

We now make an assumption which excludes an extreme, unlikely distribution of the errors:

Assumption 4.1. The local errors in modulus, i.e. $\left|e\left(\mathbf{x}_{\ell}\right)\right|$, do not coincide for all $\ell=1, \ldots, N$.

We use the representation

$$
e(s):=u_{\text {int }}(s)-u_{h}(s)=\sum_{k=1}^{N}\left(u\left(\mathbf{x}_{k}\right)-u_{h}\left(\mathbf{x}_{k}\right)\right) \varphi_{k}(s)=\sum_{k=1}^{N} e\left(\mathbf{x}_{k}\right) \varphi_{k}(s) .
$$

Proposition 4.2 Let Assumption 4.1 hold. Then there exists $\alpha>0$ such that

$$
\max _{k=1, \ldots, N}\left|u\left(\mathbf{x}_{k}\right)-u_{h}\left(\mathbf{x}_{k}\right)\right| \leq \alpha \max _{k=1, \ldots, N}\left|\int_{\Gamma_{k}}\left(\partial_{\nu_{K}} \psi_{k}\right)\left(u-u_{\text {int }}\right)\right| .
$$

Proof. Let us fix the maximal value of the l.h.s. and let it arise for index $\ell$, i.e. let $\left|e\left(\mathbf{x}_{\ell}\right)\right|=\max _{k}\left|e\left(\mathbf{x}_{k}\right)\right|:=\max _{k}\left|u\left(\mathbf{x}_{k}\right)-u_{h}\left(\mathbf{x}_{k}\right)\right|$. Using (31), we have

$$
-\int_{\Gamma_{\ell}} \partial_{\nu_{K}} \psi_{\ell}(s) e(s) d s=\sum_{k=1}^{N} e\left(\mathbf{x}_{k}\right) \int_{\Gamma_{\ell}}\left(-\partial_{\nu_{K}} \psi_{\ell}(s)\right) \varphi_{k}(s) d s .
$$

Due to the behaviour of local Green's functions and the choice of piecewise linear basis function here $\partial_{\nu_{K}} \psi_{\ell} \leq 0$ (since $\psi_{\ell} \geq 0$ and $\psi_{\ell \mid \Gamma_{\ell}}=0$ ) and $\varphi_{k} \geq 0$, hence the integrand is nonnegative, and thus we can bound the r.h.s. by factoring out the maximal value $\left|e\left(\mathbf{x}_{\ell}\right)\right|$; moreover, since not all $\left|e\left(\mathbf{x}_{k}\right)\right|$ coincide by Assumption 4.1, we obtain a strict inequality. Hence

$$
\left|-\int_{\Gamma_{\ell}} \partial_{\nu_{K}} \psi_{\ell}(s) e(s) d s\right| \leq c_{f}\left|e\left(\mathbf{x}_{\ell}\right)\right| \int_{\Gamma_{\ell}}\left(-\partial_{\nu_{K}} \psi_{\ell}(s)\right) \sum_{k=1}^{N} \varphi_{k}(s) d s .
$$

where $q=\max _{s \in \Gamma_{\ell}}\left|\sum_{1}^{N} \varphi_{k}(s) e\left(\underline{x}_{k}\right)\right| /\left|e\left(\underline{x}_{\ell}\right)\right|$.

Since $\varphi_{k}(s)$ are nonnegative weight functions and normally the sign of $e\left(\underline{x}_{k}\right)$ oscillates, we can expect $q$ to be closer to say, $1 / 2$ than to 1 .

Here, first, setting $v \equiv 1$ in (23) yields

$$
\int_{\Gamma_{\ell}}\left(-\partial_{\nu_{K}} \psi_{\ell}(s)\right)=1
$$

further, by (21), the sum $\sum_{k=1}^{N} \varphi_{k}(s)$ also equals 1 . Altogether, we obtain

$$
\left|\int_{\Gamma_{\ell}} \partial_{\nu_{K}} \psi_{\ell}(s) e(s) d s\right|<\left|e\left(\mathbf{x}_{\ell}\right)\right|
$$

hence condition (29) is satisfied and Proposition 4.1 yields the desired result. 
Remark 4.1 (i) The bound obtained in Proposition 4.2 means that the maximal nodal errors depend solely on the integrals of the local interpolation errors.

(ii) Furthermore, it is favorable that the interpolation errors in the bound appear only in integrals. Namely, whereas one always has an upper bound

$$
\left|\int_{\Gamma_{k}}\left(\partial_{\nu_{K}} \psi_{k}\right)\left(u-u_{i n t}\right)\right| \leq \max _{\Gamma_{k}}\left|u-u_{i n t}\right| \int_{\Gamma_{k}}\left|\left(\partial_{\nu_{K}} \psi_{k}\right)\right|=\max _{\Gamma_{k}}\left|u-u_{i n t}\right|
$$

(where (32) was used), on the other hand, since the interpolation error has an oscillating behaviour, it can be expected that there is an averaging (or smoothing) effect so that the errors on each $\Gamma_{k}$ can be much smaller than the maximal interpolation error, i.e. in fact

$$
\left|\int_{\Gamma_{k}}\left(\partial_{\nu_{K}} \psi_{k}\right)\left(u-u_{i n t}\right)\right| \ll \max _{\Gamma_{k}}\left|u-u_{i n t}\right| .
$$

The above method can be considered as a macro-element approximation method used as an upscaling method. For presentations of other upscaling methods see e.g. [7].

As such, it is not able to show the fine local details of the solution of a heterogeneous problem. To be able to resolve those, we must combine the method with some microelement method. As has been remarked already, this can take place by refining the mesh locally where it should give more accurate approximations.

We conclude this section with some remarks. First, if we need an accurate sampling of the heterogeneous coefficients $k$ to form the harmonic averages, we must use many integration points to compute $\bar{k}$ in (20). This can be costly. On the other hand, this initial computation can take place in parallel between the macroelements. Furthermore, for a time-dependent problem where $k$ does not depend on time, it is done just once.

Secondly, besides $[1,3,4]$, the approach of using test basis functions based on the differential equation has been applied for convection-diffusion equations with boundary layers in [6], see also references there. The coefficients in 2D problems were, however, assumed to vary locally in one direction.

Finally we remark that the method can be modified to allow for the use of a multilevel iterative solution methods. This requires, however, the computation of several levels of harmonic coefficients and will not be considered further in this paper.

\section{Concluding remarks}

A brief survey has been given on harmonic averages entering in the numerical solution of various variable coefficient problems, further, as an efficient approach related to exact difference schemes, local Green's functions used as test functions in a Petrov-Galerkin finite element method have been combined with harmonic averages.

Although exact difference schemes or use of local Green's functions may not be applicable for more general problems than we have considered, in some problems one can use them for a simpler operator $\mathcal{L}_{0}$, approximating a more general operator $\mathcal{L}$. If the highest (second) order parts of $\mathcal{L}$ and $\mathcal{L}_{0}$ are identical, then $\mathcal{L}_{0}^{-1} \mathcal{L}$ is a compact perturbation of the identity. Then, as shown in [5], a conjugate gradient method to solve $\mathcal{L} u=f$ using $\mathcal{L}_{0}$ as 
preconditioner converges with a mesh independent superlinear rate. Furthermore, since the application of the preconditioning operator $\left(\mathcal{L}_{0}\right)$ does not involve any discretization errors, the only errors that arise are due the numerical quadrature used to approximate the local residuals. This gives high accuracy of the solution at the nodal points. Note that in this way one needs only to compute local residuals, $\int_{\Omega_{l}}(\mathcal{L} u-f) v_{h} d \omega$, so there is no need to assemble a finite element matrix corresponding to $\mathcal{L}$. This can save both computational effort and need of data communication, which is important in present and future high performance computer networks with a huge number of cores.

Acknowledgement. The first author gratefully acknowledges the technical and financial support by King Abdulaziz University under grant no. 35-3-1432/HiCi. The second author gratefully thanks for the support by the Hungarian Research Grant OTKA No. K 67819.

\section{References}

[1] O. Axelsson, Stability and error estimates of Galerkin finite element approximations for convection-diffusion equations, IMA J. Numer. Anal., 1(1981), 329-345.

[2] O. Axelsson, Finite Difference Methods. Encyclopedia of Computational Mechanics, Edited by Erwin Stein, René de Borst and Thomas J.R. Hughes. 2004, John Wiley \& Sons, Ltd.

[3] O. Axelsson, E. Glushkov and N. Glushkova, The local Green's function method in singularly perturbed convection-diffusion problems. Mathematics of Computation 78 (265), 2009, 153-170.

[4] O. Axelsson, S.V. Gololobov, A combined method of local Green's functions and central difference method for singularly perturbed convection-diffusion problems. Journal of Computational and Applied Mathematics, 161, 2003, 245-257.

[5] O. Axelsson, J. Karátson, Mesh independent superlinear PCG rates via compactequivalent operators, SIAM J. Numer. Anal., 45 (2007), No.4, pp. 1495-1516.

[6] I. Babuska. G. Caloz, and E. Osborn, Special finite element methods for a class of second order elliptic problems with rough coefficients, SIAM J. Numer. Anal. 31 (1994), $945-981$.

[7] Y. Efendiev, T. Hou, T. Strinopoulos, Multiscale simulation of porous media flows in flow-based coordinate system, Comput. Geosci 12(2008), 257-272.

[8] P. W. Hemker, A numerical study of stiff two-point boundary problems, Ph.D. thesis, Mathematical Center Amsterdam, 1977.

[9] E.N. Houstis, J. R. Rice, High order methods for elliptic partial differential equations with singularities. International Journal for Numerical Methods in Engineering, Vol. 18, 1982, 737-754. 
[10] R. E. Lynch and J.R. Rice, High accuracy finite difference approximation to solutions of elliptic partial differential equations, Proc. Natl. Acad. Sci. USA, Vol. 75, No. 6, June 1978, Applied Mathematical Sciences.

[11] P. Matus, V. Irkhin and M. Lapinska-Chrzczonowicz, Exact difference schemes for time-dependent problems, Computational Methods in Applied Mathematics, 5(4), 2005, 422-448.

[12] A. Samarskii, The Theory of Difference Schemes, Marcel Dekker, Inc. New York, Basel, 2001. 